\title{
Medial Approach to the Sciatic Nerve at the Popliteal Fossa in the Supine Position with Ultrasound Guidance and Nerve Stimulator
}

\author{
Tomoaki Yanaru, Kenji Shigematsu, Kazuo Higa, Erisa Nakamori, and Keiichi Nitahara \\ Department of Anesthesiology, Faculty of Medicine, Fukuoka University, 45-1, 7-Chome, Nanakuma, Jonan-ku, \\ Fukuoka 814-0180, Japan \\ Correspondence should be addressed to Tomoaki Yanaru, yanaru@fukuoka-u.ac.jp
}

Received 2 January 2012; Accepted 29 January 2012

Academic Editors: D. E. Selander and C.-T. Wu

Copyright ( $\odot 2012$ Tomoaki Yanaru et al. This is an open access article distributed under the Creative Commons Attribution License, which permits unrestricted use, distribution, and reproduction in any medium, provided the original work is properly cited.

\begin{abstract}
Ultrasound guided sciatic nerve block (SNB) at the popliteal fossa is performed with the lateral approach in the supine position or with the lateral or posterior approach in the prone position. When the sciatic nerve (SN) is blocked with the lateral approach in the supine position, the lower limb must be sufficiently elevated to enable adequate space around the knee joint for transducer application. When the $\mathrm{SN}$ is blocked in the prone position, the patients' position needs to be changed. We report a medial approach to the SNB at the popliteal fossa in the supine position with ultrasound guidance. Ten patients scheduled for elective knee or foot surgery participated in this study. Patients were placed in the supine position, with the hip and knee on the operated side flexed and the thigh externally rotated at approximately 45 degrees. A block needle was inserted in-plane with the transducer toward the $\mathrm{SN}$ bifurcation from the medial side of the thigh. The block performance time for SNB was $1.8 \pm 0.5 \mathrm{~min}$ (1.3-3.1 min). All blocks were effective. Our medial approach to the SN in the supine position with ultrasound guidance does not require elevation of the patient's lower limb or a change in the patient's position.
\end{abstract}

\section{Introduction}

Sciatic nerve block (SNB) at the popliteal fossa is used for surgery below the knee level [1], usually with femoral nerve block (FNB) or other blocks [1], depending on the surgical sites. Ultrasound guided SNB in the popliteal fossa is performed with the lateral approach in the supine position $[2,3]$, or with the lateral or posterior approach in the prone position $[4,5]$. When the sciatic nerve (SN) is blocked with the lateral approach in the supine position, the lower limb must be sufficiently elevated to enable adequate space around the knee joint for transducer application $[2,3]$. When the SN is blocked in the prone position, patients require their position to be changed for additional FNB and/or surgery [4, 5].

We investigated a medial approach to block the $\mathrm{SN}$ at the popliteal fossa in the supine position with ultrasound guidance. This approach did not need elevation of the lower limb or a position change. A medial approach to block the SN at the popliteal fossa in the supine position with a nerve stimulator was described [6]. This is the first report of ultrasound guided SNB at the popliteal fossa with the medial approach in the supine position.

\section{Materials and Methods}

This study was approved by the Ethics Committee of Fukuoka University Hospital. Ten patients scheduled for elective knee or foot surgery gave their written informed consent to participate in this study.

In the operating room, each patient received midazolam $2 \mathrm{mg}$ and fentanyl $50 \mu \mathrm{g}$ intravenously. SNB was performed first, and FNB was subsequently performed. Patients were placed in the supine position, with the hip and knee on the operated side flexed and the thigh externally rotated at approximately 45 degrees (Figure 1). Peripheral nerve blocks were performed with the aid of M-Turbo Ultrasound 


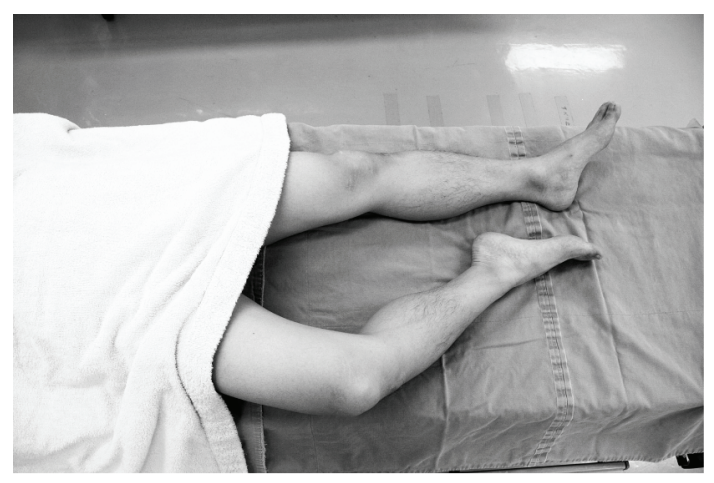

FIGURE 1: The patient is placed in the supine position with the hip and knee flexed and the thigh externally rotated at approximately 45 degrees.

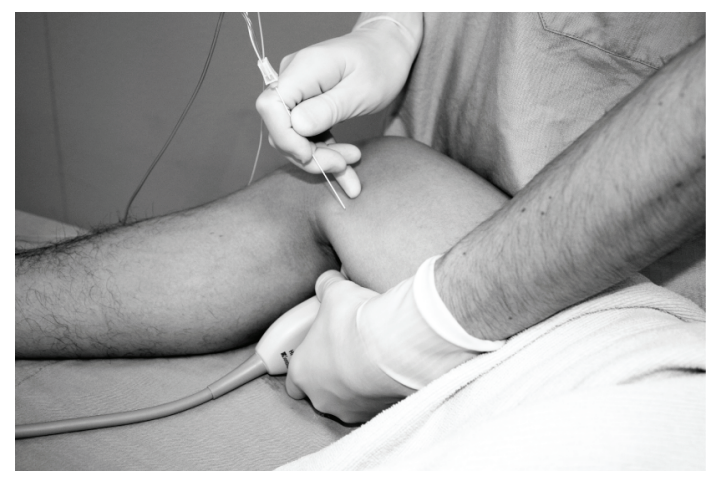

FIGURE 2: The block needle is inserted in-plane with the transducer toward the sciatic nerve bifurcation from medial side of the thigh.

Machine (Sonosite, Bothell, WA, USA), with a linear 6 to $15 \mathrm{MHz}$ transducer (HFL-50x, Sonosite). The popliteal vessels and the tibial nerve were identified first by positioning the transducer in the popliteal fossa. The transducer was then slid proximally and positioned at the level of the SN bifurcation to locate the tibial nerve and the common peroneal nerve. A 21-gauge, $100 \mathrm{~mm}$ needle (Stimuplex A, B. Braun Melsunger AG, Germany), which was connected to a nerve stimulator (Stimuplex DIG RC, B. Braun Melsunger $A G)$, was inserted in-plane with the transducer toward the $\mathrm{SN}$ bifurcation from the medial side of the thigh (Figure 2). The nerve stimulator was set to a pulse duration of $0.1 \mathrm{~ms}$, current intensity $0.5 \mathrm{~mA}$, and stimulating frequency of $2 \mathrm{~Hz}$. When the needle reached the point eliciting foot movement, $0.5 \%$ ropivacaine was injected around the nerve in $5 \mathrm{~mL}$ increments, after negative aspiration test for blood, to a total volume of $20 \mathrm{~mL}$.

Ultrasound guided FNB was then performed at the inguinal groove in the same supine position. The needle was inserted in-plane with the transducer from the lateral side of the inguinal groove. The nerve stimulator was set to be the same pulse duration, current intensity, and stimulating frequency as that for the SNB. When the needle reached the point eliciting patellar movement, $0.5 \%$ ropivacaine was
TABle 1: Demographic data of the patients.

\begin{tabular}{|c|c|}
\hline Age (yr) & $53 \pm 18$ \\
\hline Height $(\mathrm{cm})$ & $161 \pm 10$ \\
\hline Weight (kg) & $62 \pm 12$ \\
\hline BMI $\left(\mathrm{kg} / \mathrm{m}^{2}\right)$ & $24 \pm 3$ \\
\hline $\operatorname{Sex}(M / F)$ & $4 / 6$ \\
\hline ASA status (I/II) & $6 / 4$ \\
\hline \multicolumn{2}{|l|}{ Operation $(n)$} \\
\hline Knee arthroscopy & 5 \\
\hline Foot or ankle surgery & 5 \\
\hline
\end{tabular}

BMI: body mass index.

ASA: American Society of Anesthesiologists.

$($ Mean $\pm \mathrm{SD})$.

injected around the nerve in $5 \mathrm{~mL}$ increments, after negative aspiration test for blood, to a total volume of $20 \mathrm{~mL}$.

The operation was started after hypoesthesia at the regions innervated by the blocked nerves was confirmed. Patients were sedated with a bolus injection of propofol 40$50 \mathrm{mg}$ followed by continuous infusion of propofol 3-4 mg/ $\mathrm{kg} / \mathrm{h}$. Fentanyl $50 \mu \mathrm{g}$ was administrated to supplement nerve blocks, with a total of 100 to $200 \mu \mathrm{g}$.

Measurements included the following: block performance time for SNB, which we defined as the duration of time from insertion of the block needle until completion of injection of ropivacaine; block performance time for both SNB and FNB, which we defined as the time elapsed from the insertion of the block needle for the SNB until the withdrawal of the needle for the FNB; foot movement elicited by nerve stimulation; onset time of sensory blocks.

Cold sensation was evaluated with an alcohol swab every 5 min after injection of ropivacaine at the plantar aspect of the foot, which is innervated by the tibial nerve, and the dorsal aspect of the foot is innervated by the common peroneal nerve.

Continuous variables are presented as mean \pm SD. Data were assessed by the Student's $t$-test. Statistical significance was considered as $P<0.05$.

\section{Results}

The demographic data of the patients are summarized in Table 1. All patients were successfully placed in position for the block.

The block performance time for SNB was $1.8 \pm 0.5 \mathrm{~min}$ (1.3-3.1 $\mathrm{min}$ ), and the block performance time for both SNB and FNB was $3.7 \pm 0.8 \mathrm{~min}(3.0-5.9 \mathrm{~min})$. The foot moved downward in response to electrical stimulation in all the patients. The onset of sensory blockade was $7.0 \pm 2.6 \mathrm{~min}(5-$ $10 \mathrm{~min})$ in the tibial nerve area and $6.5 \pm 2.4 \mathrm{~min}(5-10 \mathrm{~min})$ in the common peroneal nerve area. There was no significant difference in the onset of sensory block between the tibial and common peroneal nerve areas. The time from end of the block to the beginning of surgery was $20 \pm 8 \mathrm{~min}$. The duration of operation was $49 \pm 29$ min. 
All blocks were effective. There was no need to change anesthetic management in any of the patients.

\section{Discussion}

We report a medial approach to the sciatic nerve at the popliteal fossa in the supine position with ultrasound guidance. It was easy to perform under ultrasound guidance and did not need any position changes. Our medial approach to the SN in the supine position did not require elevation of the patient's lower limb or a change in the patient's position. However, since our method requires the hip and knee to be flexed and the thigh externally rotated at approximately 45 degrees (Figure 1), this approach is difficult for patients who have movement restrictions of the hip and/or knee joints.

Guntz et al. [6] reported that the block performance time of SNB with a nerve stimulator with the medial approach in the supine position was $100 \mathrm{sec}$ (range 55-165 sec). Buys et al. [3] reported that the block performance time of ultrasound guided SNB with the lateral approach in the supine position, which was defined as the time elapsed from the insertion of the block needle until the completion of local anesthetic injection, was $2.9 \pm 1.7$ (mean \pm SD) min. Prasad et al. [5] reported that the block performance time of ultrasound guided SNB with the lateral approach in the prone position, which was defined as the time elapsed from ultrasound transducer preparation until the completion of anesthetic injection, was $9 \pm 3$ (mean $\pm S D$ ) min. In our study, the block performance time of SNB, which we defined as the time from insertion of the block needle until completion of injection of ropivacaine, was only $1.8 \pm 0.5 \mathrm{~min}$, and the block performance time for both SNB and FNB was $3.7 \pm 0.8 \mathrm{~min}$.

The tibial and common peroneal nerves bifurcate from the SN. The tibial nerve travels down the center of the thigh, and the common peroneal nerve runs along the lateral side of the thigh [6]. When the block needle is inserted below the SN bifurcation from the medial side of the thigh in the supine position, the tip of the needle may be closer to the tibial nerve than the common peroneal nerve. Electrical stimulation of the tibial nerve causes plantar flexion, and in the common peroneal nerve it causes dorsal flexion. Our patients showed plantar flexion in response to electrical stimulation, indicating that the tibial nerve was stimulated in all patients.

When the SN is blocked with the lateral approach in the supine position, the onset of block, as assessed by pin prick, is faster in the area innervated by the common peroneal nerve than that by the tibial nerve [3]. In our study, there was no significant difference in the onset of sensory block between the tibial and common peroneal nerves. The tip of the needle in the medial approach may be closer to the tibial nerve than in lateral approach.

We have described a medial approach to the $\mathrm{SN}$ at the popliteal fossa in the supine position with ultrasound guidance. There was no difference in performance time in our method compared with previous reports of medial approach with a nerve stimulator in the supine position, the lateral approach to the $\mathrm{SN}$ in the supine position with ultrasound guidance, or the lateral approach in the prone position with ultrasound guidance; however, our method did not require elevation of the patient's lower limb or a change in position.

\section{References}

[1] M. K. Karmakar, W. H. Kwok, A. M. Ho, K. Tsang, P. T. Chui, and T. Gin, "Ultrasound-guided sciatic nerve block: description of a new approach at the subgluteal space," British Journal of Anaesthesia, vol. 98, no. 3, pp. 390-395, 2007.

[2] C. J. L. McCartney, I. Brauner, and V. W. S. Chan, "Ultrasound guidance for a lateral approach to the sciatic nerve in the popliteal fossa," Anaesthesia, vol. 59, no. 10, pp. 1023-1025, 2004.

[3] M. J. Buys, C. D. Arndt, F. Vagh, A. Hoard, and N. Gerstein, "Ultrasound-guided sciatic nerve block in the popliteal fossa using a lateral approach: onset time comparing separate Tibial and common peroneal nerve injections versus injecting proximal to the bifurcation," Anesthesia and Analgesia, vol. 110, no. 2, pp. 635-637, 2010.

[4] A. Sinha and V. W. S. Chan, "Ultrasound imaging for popliteal sciatic nerve block," Regional Anesthesia and Pain Medicine, vol. 29, no. 2, pp. 130-134, 2004.

[5] A. Prasad, A. Perlas, R. Ramlogan, R. Brull, and V. Chan, "Ultrasound-guided popliteal block distal to sciatic nerve bifurcation shortens onset time: A prospective randomized doubleblind study," Regional Anesthesia and Pain Medicine, vol. 35, no. 3, pp. 267-271, 2010.

[6] E. Guntz, P. Herman, E. Debizet, D. Delhaye, V. Coulic, and M. Sosnowski, "Sciatic nerve block in the popliteal fossa: description of a new medial approach," Canadian Journal of Anesthesia, vol. 51, no. 8, pp. 817-820, 2004. 


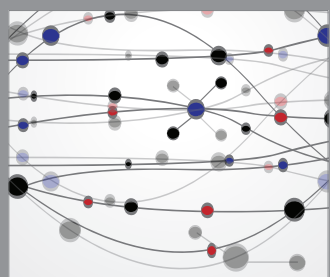

The Scientific World Journal
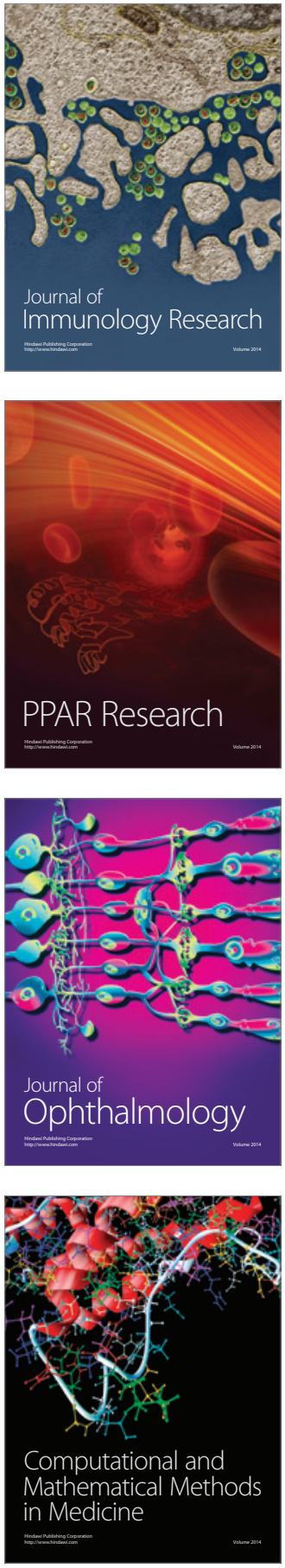

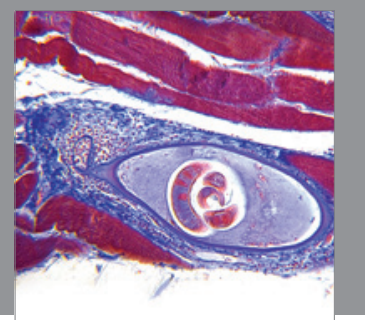

Gastroenterology

Research and Practice
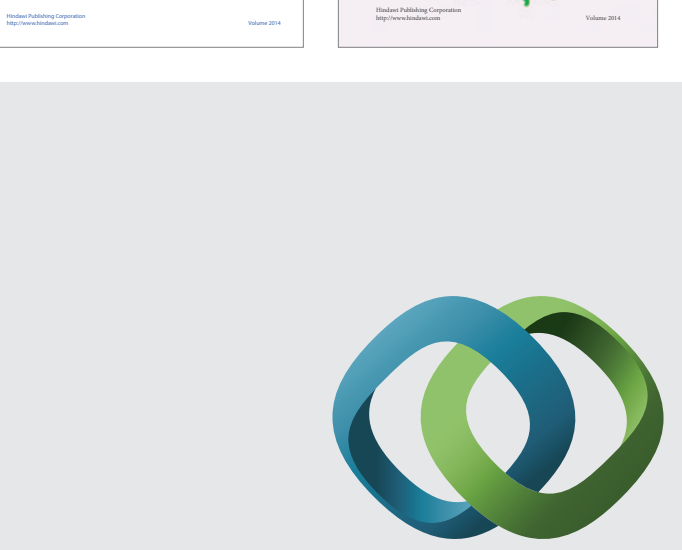

\section{Hindawi}

Submit your manuscripts at

http://www.hindawi.com
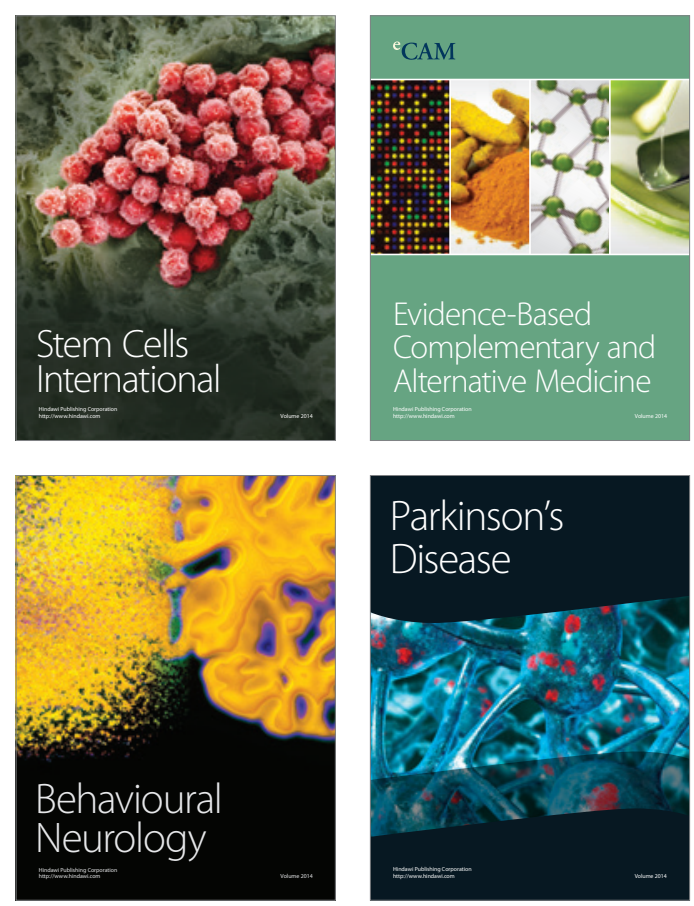

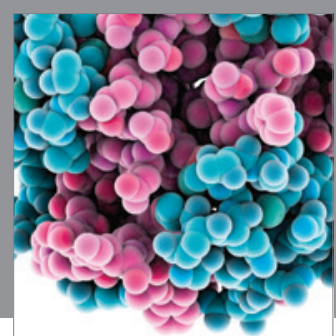

Journal of
Diabetes Research

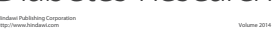

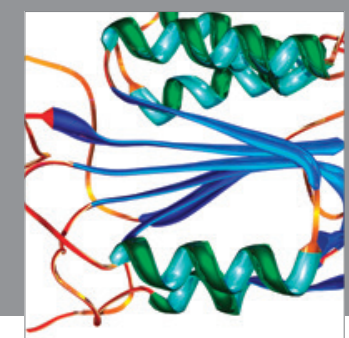

Disease Markers
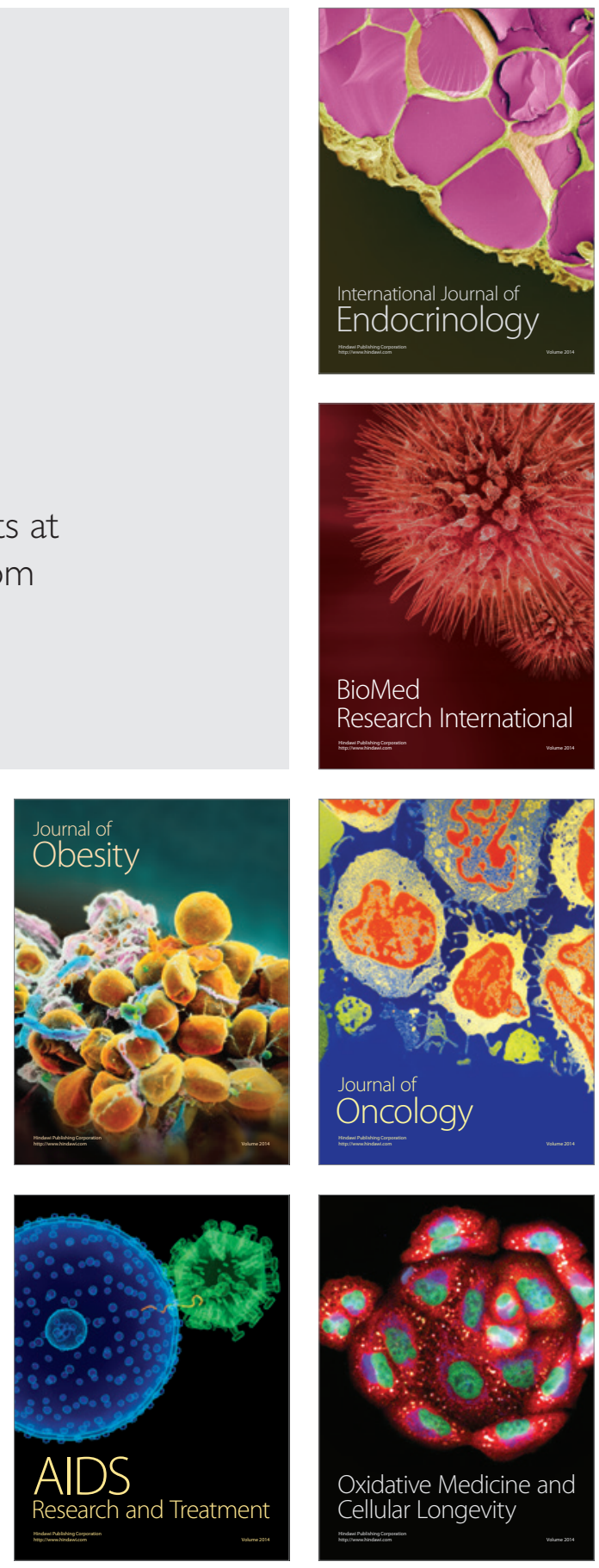